\title{
Novel educational approach to some aspects of optical information processing
}

Prasanta Ghosh, Partha Banerjee, P. Berra

Prasanta K. Ghosh, Partha P. Banerjee, P. Bruce Berra, "Novel educational approach to some aspects of optical information processing," Proc. SPIE 1603, Education in Optics, (1 March 1992); doi: 10.1117/12.57870 


\title{
A novel educational approach to some aspects of optical information processing
}

\author{
P.K. Ghosh, P.P. Banerjee and P.B. Berra \\ Department of Electrical and Computer Engineering \\ Syracuse University, Syracuse, New York 13244-1240
}

\section{INTRODUCTION}

Faculty members involved in the optics program in the Department of Electrical and Computer Engineering at Syracuse University have diverse research interests. We use this diversity in our teaching methods/materials to educate future engineers. Our instructional methods, both classroom and laboratory, are designed to provide physical insight of optical devices and systems. Our electro-optics laboratory is equipped with three optical benches, four helium neon lasers, a Nd:YAG laser, optical peripheral equipment, a variety of acousto-optic signal/image processing set up, and related electronic equipment of reasonable sophistication.

We have offered several graduate level courses primarily based on recent research. These courses cover various aspects of optics namely Fourier optics, nonlinear optics, quantum electronics, electro-optic integrated circuits and devices, fiber optic, etc. In addition, we have offered a course in optical database machines. In the future we will offer a undergraduate course in applied optics. In what follows, we will describe, in detail, some of the approaches we have developed which help students to gain physical understanding of complicated optical physics, including illustrative classroom demonstration of optical processing and the realization of optical logic operations.

\section{NOVEL APPROACH TO CLASS-ROOM TEACHING OF VARIOUS CONCEPTS IN OPTICS}

\subsection{Transfer-Function Approach to Diffraction}

To find the exact nature of the amplitude and/or phase distribution during the propagation of a diffracted light beam one can either employ the eikonal (ray) equations to find the amplitude and phase distribution individually, or else find a way to predict the complex amplitude all at once. The transfer-function approach, we believe, does that and also is easy to compute.

The development of this method starts with the assumption that the total wavefunction $\psi(x, y, z, t)$ comprises a complex envelope $\psi_{c}(x, y, z)$ riding on a carrier frequency $\omega_{0}$ and propagation constant $k_{0}$ that propagates in the $+z$ direction:

$$
\psi(x, y, z, t)=\operatorname{Re}\left\{\psi_{0}(x, y, z) \exp \left[j\left(w_{0} t-k_{0} z\right)\right]\right\}, \frac{\omega_{0}}{k_{0}}=v .
$$

Now, starting from the wave equation in cartesian coordinates and assuming the $\psi_{e}(x, y, z)$ is slowly varying function of $\mathrm{z}$ one can obtain the paraxial wave equation,

$$
2 j k_{0} \frac{\partial \Psi_{0}}{\partial z}=\frac{\partial^{2} \Psi_{0}}{\partial x^{2}}+\frac{\partial^{2} \Psi_{0}}{\partial y^{2}}
$$

The above equation describes the propagation of the envelope starting from an initial profile $\psi_{c}(x, y, 0)$. The solution of Eq. (2) in the Fourier domain is

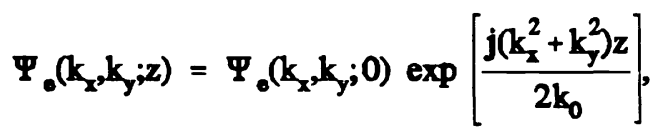

where,

$$
\Psi_{e}\left(k_{x}, k_{y} ; z\right)=F_{x y}\left\{\Psi_{e}(x, y, z)\right\}
$$


For notational simplicity, we will call $\Psi_{c}\left(k_{\alpha}, k_{y} ; 0\right)=\Psi_{\infty 0}\left(k_{x}, k_{y}\right)$. We can interpret Eq. (3) in the following way: Consider a linear system with $\Psi_{c 0}\left(\mathrm{k}_{\mathrm{x}}, \mathrm{k}_{\mathrm{y}}\right)$ as its input spectrum (i.e., at $\mathrm{z}=0$ ) and where the output spectrum is $\Psi_{\mathrm{e}}\left(\mathrm{k}_{\mathrm{x}}, \mathrm{k}_{\mathrm{y}} ; \mathrm{z}\right)$. Then the spatial frequency response of the linear system is given by

$$
\frac{\Psi_{\bullet}}{\Psi_{\infty}} \Delta H\left(k_{x}, k_{y} ; z\right)=\exp \left[\frac{j\left(k_{x}^{2}+k_{j}^{2}\right) z}{2 k_{0}}\right] .
$$

We call $\mathrm{H}\left(\mathbf{k}_{x}, \mathbf{k}_{\mathbf{y}} ; \mathrm{z}\right)$ the spatial transfer function of propagation of light through a distance $\mathrm{z}$ in the medium as shown in Fig. 1.

Taking the inverse Fourier transform one obtains:

$$
\begin{aligned}
\Psi_{\odot}(x, y, z) & =\Psi_{\infty 0}(x, y) * h(x, y, z) \\
& =\frac{j k_{0}}{2 \pi z} \int_{-\infty}^{\infty} \int_{-\infty}^{-} \Psi_{\infty}\left(x^{\prime}, y^{\prime}\right) \\
& \exp \left[-j \frac{k_{0}}{2 z}\left\{\left(x-x^{\prime}\right)^{2}+\left(y-y^{\prime}\right)^{2}\right\}\right] d x^{\prime} d y^{\prime}
\end{aligned}
$$

where * denotes convolution. The way Eq. (5) has been written indicates that the tranverse coordinates are $x^{\prime}, y^{\prime}$ in the input plane and $x, y$ in the output plane at a distance $z$ away from the input plane (see Fig. 1).

Eq. (5) is termed the Fresnel diffraction formula and describes the Fresnel diffraction of a beam during propagation and having an arbitrary initial complex amplitude profile.

Using the above mentioned approach it is easy to visualize the nature of a diffracted Gaussian beam as follows. Let

$$
\Psi_{\infty}(x, y)=\exp \left[-\frac{\left(x^{2}+y^{2}\right)}{w_{0}^{2}}\right]
$$

then

$$
\begin{aligned}
& \Psi_{0}\left(k_{x}, k_{y} ; z\right)=\Psi_{\infty 0}\left(k_{x}, k_{y}\right) H\left(k_{x}, k_{y} ; z\right) \\
&= \pi w_{0}^{2} \exp \left[-\frac{\left(k_{x}^{2}+k_{y}^{2}\right) w_{0}^{2}}{4}\right] \exp \left[\frac{j\left(k_{x}^{2}+k_{j}^{2}\right) z}{2 k_{0}}\right] \\
&=\pi w_{0}^{2} \exp \left[\frac{j\left(k_{x}^{2}+k_{y}^{2}\right) q}{2 k_{0}}\right],
\end{aligned}
$$

where,

$$
q \Delta z+\frac{j k_{0} w_{0}^{2}}{2} \Delta z+j z_{R}
$$

is called the $q$ parameter of the Gaussian beam and $z_{R}$ is the Rayleigh length. The propagation of a Guassian beam is completely described by the transformation of its q parameter. The inverse Fourier transform of Eq. (6) provides the nature of the Gaussian beam during propagation, 


$$
\begin{aligned}
\downarrow_{0}(x, y, z) & =\frac{j k_{0} w_{0}^{2}}{2 q} \exp \left[-\frac{j\left(x^{2}+y^{2}\right) k_{0}}{2 q}\right] \\
& =\left(\frac{w_{0}}{w(z)}\right) \exp \left\{-\frac{x^{2}+y^{2}}{w^{2}(z)}\right\} \\
& \cdot \exp \left\{-\frac{j k_{0}}{2 R}\left(x^{2}+y^{2}\right)\right\} \exp (-j \phi),
\end{aligned}
$$

where,

$$
w^{2}(z)=\frac{2 z_{R}}{k_{0}}\left[1+\left(\frac{z}{z_{r}}\right)^{2}\right], \quad R(z)=\frac{z^{2}+z_{R}^{2}}{z}, \phi(z)=-\tan ^{-1}\left(\frac{z}{z_{R}}\right) .
$$

This result indicates, as expected, a decrease in amplitude during propagation and an increase in its width w (initially equal to the waist size $w_{0}$ ). Also, one can easily evaluate the Rayleigh length, phase curvature, the position of the minimum radius of curvature, etc. Other examples of Fresnel diffraction using the transfer function approach may be found in Ref. [1].

\subsection{Transfer Function Approach to Holographic Reconstruction}

The above transfer function concept can also be used to understand the nature of holographic image during reconstruction [1]. When a Leith-Upatneiks hologram is illuminated by a collimated beam, as shown in Fig. 2, the expression for the optical field immediately behind the hologram will contain a term proportional to $\psi_{0 A}(x, y) \exp \left[+j k_{0}(\sin \theta) y\right.$, which is obtained from the amplitude transmittance of the Leith-Upatneiks hologram, where $\psi_{0 \mathrm{~A}}$ is the complex field due to the object $t(x, y)$ on the plane of the film where the hologram is recorded. Written explicitly, this term reads

$$
\begin{gathered}
\phi_{\mathrm{m} d}=-j \frac{k_{0}}{2 \pi \mathrm{z}} \exp \left[-j \mathrm{k}_{0}(\mathrm{z}-\mathrm{y} \sin \theta)\right] \\
\cdot \int_{-\infty}^{-} \int_{-\infty}^{-} t^{*}\left(x^{\prime}, y^{\prime}\right) \exp \left\{\frac{+j k_{0}}{2 z}\left[\left(x-x^{\prime}\right)^{2}+\left(y-y^{\prime}\right)^{2}\right]\right\} d x^{\prime} d y^{\prime}
\end{gathered}
$$

Application of the transfer function concept readily allows one to realize that this complex field Eq. (8) gives rise to a real image, as follows. The Fourier transform of Eq. (8) is proportional to

$$
T^{*}\left(-k_{2},-k_{j}\right) \exp \left[-j\left(k_{\alpha}^{2}+k_{j}^{2}\right) z / 2 k_{0}\right]
$$

where we have disregarded the exponential before the integral for simplicity. Now, after propagation through a distance $z^{\prime}$, the Fourier transform of the complex field must be

$$
T^{*}\left(-k_{x},-k_{\gamma}\right) \exp \left\{-j\left[\left(k_{x}^{2}+k_{j}^{2}\right) / 2 k_{0}\right]\left(z-z^{\prime}\right)\right\}
$$

When $z^{\prime}=z$, the complex field, therefore, will be $t^{*}(x, y)$, and hence the intensity will be proportional to $|t(x, y)|^{2}$, indicating a real image. 


\subsection{Transfer-Function Approach to Acousto-optic Interactions}

The Fourier-transform technique also enables us to define and write down explicit expressions for the effective spatial interaction transfer functions relating the output angular plane wave spectra of the scattered orders to the input angular plane wave spectrum during acousto-optic interaction. To demonstrate this approach we start from the wave equation for a component of the electric field (assumed transverse) in a medium with permeability $\mu_{0}$ and whose permittivity is given as $\varepsilon(x, y)=\varepsilon_{0}+$ $\varepsilon^{\prime}(\mathrm{x}, \mathrm{z})$, where $\varepsilon^{\prime}$ is a "slowly-varying" function of $\mathrm{x}$ and $\mathrm{z}$ :

$$
\psi_{u}-c_{0}^{2} \nabla^{2} \psi-\left(\varepsilon^{\prime} / \varepsilon_{0}\right) \psi_{u} ; c_{0}^{2}=1 /\left(\mu_{0} \varepsilon_{0}\right)
$$

The variation of $\varepsilon^{\prime}$ with respect to $\mathrm{x}, \mathrm{z}$ is due to a propagating sound wave $\mathrm{s}(\mathrm{x}, \mathrm{z})$. In fact, $\varepsilon^{\prime}$ may be explicitly written as

$$
\varepsilon^{\prime}(x, z)=\varepsilon_{0} C \operatorname{Re}\left\{S_{0}(x, z) \exp \left\{j\left(\Omega_{0} t-K_{0} x\right)\right\}\right\}
$$

where $\mathrm{C}$ denotes an interaction constant and the rest of the expression on the right hand side of Eq. (12) is the representation of the sound field propagating (dominantly) in the $+x$ direction (see Fig. 3). Now, the simple heuristic treatment of acoustooptic interactions connotes to the creation of several scattered orders, each separated from the next by an angle equal to twice the Bragg angle. We may thus represent the scattered orders $\psi_{\mathrm{m}}$ due to acoustooptic interaction mathematically by writing

$$
\begin{aligned}
\psi(x, y, z) & =\sum_{m}^{\Sigma} \operatorname{Re}\left\{\psi_{\operatorname{mom}}(x, y)\right. \\
\cdot & \left.\exp \left\{j\left(\left(w_{0}+m \Omega_{0}\right) t-k_{a \max } x-k_{\max _{x}} z\right)\right\}\right\}
\end{aligned}
$$

where $k_{o m x}, k_{o m z}$ define the direction of propagation $\phi_{m}$ of each scattered order, with

$$
k_{\mathrm{omx}}=k_{0} \sin \phi_{m} ; k_{o m z}=k_{0} \cos \phi_{m}
$$

and

$$
\sin \phi_{m}=\sin \phi_{\mathrm{mc}}+\mathrm{mK}_{\mathrm{m}} / \mathrm{k}_{0}
$$

$\phi_{\text {inc }}$ denotes the incident angle of the optical field entering the acoustooptic interaction region. If we now put Eqs. (12) and (13) in Eq. (11) and gather the coefficients of exp $j\left(\left(\omega_{0}+m \Omega_{0}\right) t\right)$, we obtain, after considerable algebra,

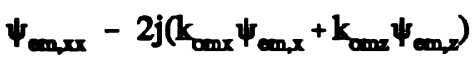

$$
\begin{aligned}
& +\left(k_{0}^{2} C / 2\right)\left[S_{e}(x, z) \Psi_{\alpha(m-1)} \exp \left\{-j\left(k_{\alpha(m-1) z}-k_{\alpha m z}\right) z\right\}\right. \\
& \left.+S_{0}^{*}(x, z) \Psi_{\alpha(m+1)} \exp \left\{-j\left(k_{\alpha(m+1) z}-k_{\alpha m z}\right) z\right\}\right]=0
\end{aligned}
$$

In deriving the Eq. (15), an assumption $\left|k_{0 z} \psi_{a z}\right| \gg\left|\Psi_{e, z z}\right|$ for every diffracted order, was made and a term $\left(\mathrm{k}_{0 \mathrm{~m}}^{2}-\mathrm{k}_{0}^{2}\right) \Psi_{\mathrm{em}}$ was neglected on the basis that $\left(\mathrm{k}_{\mathrm{om}}^{2}-\mathrm{k}_{0}^{2}\right)$ only changes the amplitude and not the phase of $\psi_{\mathrm{em}}$.

The first term on the left hand side of Eq. (15) represents the effect of propagational diffraction. The presence of $\mathrm{k}_{0 \max }$, $\mathbf{k}_{\mathrm{ome}}$ in the second term defines the direction of propagation of the $\mathbf{m}$-th order scattered light. Finally, the last terms describe the scattering of the light by the sound. 
In the traditional treatment ${ }^{2}$, the first term is neglected on the basis of the assumption that the incident and scattered light are plane waves. Also, the sound waves are assumed to be quasi-planar in a bounded sound column. Furthermore, by neglecting the term $-2 \mathrm{jk}_{\mathrm{omx}} \Psi_{\mathrm{em}, x}$, in Eq. (15), the coupled equation between the various scattered orders are written as

$$
\begin{gathered}
\psi_{c m z}=-j\left[D \psi_{\alpha(m+1)}+E \psi_{\alpha(m-1)}\right], \\
D=\left(k_{0} C A^{*} / 4\right) \exp \left\{-j k_{0} z\left(\cos \phi_{m+1}-\cos \phi_{m}\right)\right\}, \\
E-\left(k_{0} C A / 4\right) \exp \left\{-j k_{0} z\left(\cos \phi_{m-1}-\cos \phi_{m}\right)\right\},
\end{gathered}
$$

where Eq. (14a) has been used to express the $k_{0 \mathrm{~m}}{ }^{\prime} \mathrm{s}$ in terms of $\phi_{\mathrm{m}}{ }^{\prime} \mathrm{s}$, and where a sound field amplitude $s_{\mathrm{e}}(\mathrm{x}, \mathrm{z})=\mathrm{A}$ has been assumed in the bounded sound column. A special case of the above interaction is Bragg diffraction, which is characterized by the generation of two scattered order as shown in Fig. 4. Referring to Eq. (16a) we find that for the diffracted orders 0 and -1 (downshifted interaction), the coupled equations read

$$
\frac{d \phi_{0}}{d z}=\frac{-j k_{0} C A}{4 \cos \phi_{0}} \psi_{-1} \exp \left[-j k_{0} z\left(\cos \phi_{-1}-\cos \phi_{0}\right)\right]
$$

and

$$
\frac{d \phi_{-1}}{d z}=\frac{-j k_{0} C A^{*}}{4 \cos \phi_{-1}} \psi_{0} \exp \left[+j k_{0} z\left(\cos \phi_{-1}-\cos \phi_{0}\right)\right]
$$

where we have neglected the subscript " $\mathrm{e}$ " for notational convenience. Assuming phase synchronism between the 0 th and $-1 s t$ orders, one must impose $\cos \phi_{-1}=\cos \phi_{0}$, implying

$$
\phi_{0}=-\phi_{-1}=\phi_{\mathrm{bc}}=\phi_{\mathrm{B}}
$$

as $\phi_{-1} \neq \phi_{0}$ because different scattered orders must exit at different angles. Thus, the 0 th and diffracted (-1st) order propagates symmetrically with respect to the sound wavefronts. We, then, can write the coupled equations describing the downshifted interaction as

$$
\begin{aligned}
& \frac{d \psi_{0}}{d z}=\frac{-j k_{0} C A}{4 \cos \phi_{B}} \psi_{-1} \\
& \frac{d \psi_{-1}}{d z}=\frac{-j k_{0} C A^{*}}{4 \cos \phi_{B}} \Psi_{0}
\end{aligned}
$$

where $-j \mathrm{k}_{0} \mathrm{CA} / 4 \cos \phi_{\mathrm{B}}$ and $-\mathrm{jk} \mathrm{CA}_{0} \mathrm{CA}^{*} / 4 \cos \phi_{\mathrm{B}}$ are the coupling coefficients. Similar coupled equations can be written for the upshifted interaction. Taking into account the following boundary condition

$$
\psi_{m}=\Psi_{\text {mec }} \delta_{\text {mo }} \text { at } z \leq 0 \text {, }
$$

where $\delta_{\mathrm{m} 0}$ represents the Kronecker delta function, and with $\phi_{\mathrm{inc}} \ll 1$, the solution of the ordinary differential equations eqs. $(19,20)$ is 


$$
\begin{gathered}
\psi_{0}=\Psi_{\text {mc }} \cos \left(\frac{\hat{Q}}{2}\right), \\
\psi_{-1}=-\mathrm{j} \frac{A^{*}}{|A|} \Psi_{\text {mc }} \sin \left(\frac{\hat{Q}}{2}\right),
\end{gathered}
$$

where $\hat{\alpha}=k_{0} C|A| L / 2$, for the downshifted interaction, and are the well-known expressions for the scattered light in Bragg diffraction.

In what follows, we will take Eq. (15) as a starting point and Fourier-transform the equation to find the evolution of the angular orders. This gives

$$
\begin{aligned}
& \Psi_{e m 2}=j\left[\left(k_{x}^{2}+2 k_{x} k_{0 x m}\right) /\left(2 k_{o m m}\right)\right] \Psi_{o(m-1)} \\
& -j D \Psi_{\alpha(m+1)}-j E \Psi_{\alpha(m-1)},
\end{aligned}
$$

where D and E are defined in Eqs. $(16 \mathrm{~b}, 16 \mathrm{c})$ and $\Psi_{\mathrm{em}}$ is the Fourier transform of $\Psi_{\mathrm{em}}$. We have taken the amplitude of the sound field to be constant, assuming once again, a bounded column. In what follows, we will examine the scattering of an arbitrary light wave profile incident on the acoustooptic cell at Bragg incidence $\left(\phi_{\text {inc }}=\phi_{\mathrm{B}}\right)$. In this configuration only the zero and minus one scattered orders remian Fig. 5. The coupled equations (23) then reduce to

$$
\begin{aligned}
& \Psi_{\infty, z}=j\left[k_{x}^{2}+2 k_{x} k_{\infty a d} /\left(2 k_{\infty+\infty}\right)\right] \Psi_{\infty}-j E \Psi_{\alpha-1)} \\
& \Psi_{\alpha(-1) \Sigma}=j\left[\left(k_{x}^{2}+2 k_{x} k_{\alpha \alpha(-1)} /\left(2 k_{\alpha \alpha(-1)}\right)\right] \Psi_{\alpha(-1)}-j D \Psi_{\infty}\right.
\end{aligned}
$$

since $\phi_{0}=\phi_{\mathrm{B}}, \phi_{-1}=\phi_{0}-2 \phi_{\mathrm{B}}=-\phi_{\mathrm{B}}$, the D and $\mathrm{E}$ in $16 \mathrm{~b}$ and $16 \mathrm{c}$ become

$$
\begin{aligned}
& D=k_{0} C A^{*} / 4 \\
& E=k_{0} C A / 4
\end{aligned}
$$

and

$$
\begin{aligned}
k_{0 x 0} & =k_{0} \sin \phi_{B} \\
k_{\infty 00} & =k_{0} \cos \phi_{B} \\
k_{0 x(-1)} & =-k_{0} \sin \phi_{B} \\
k_{\text {od }(-1)} & =k_{0} \cos \phi_{B} .
\end{aligned}
$$

Our intention now will be to derive transfer functions relating the output spectra of the scattered orders to the input spectrum of the arbitrary beam profile. It will be convenient to track these relationships along $z$ ' and $z "$, which are the nominal directions of propagation of the zero and the minus one order, as shown in Fig. 4. This will enable us to define what we call the 
interaction transfer functions in the presence of propagational diffraction. The interaction transfer functions describe the output optical fields in the zero and minus one orders, along their nomonal directions of propagation, in terms of the input optical field. For the purpose of deriving the interaction transfer function for the zero order optical field we convert Eqs. $(24,25)$ to a new coordinate system $\left(x^{\prime}, z^{\prime}\right)$ and the resulting Fourier-transform is

$$
\begin{gathered}
\Psi^{\prime}\left(k_{x^{\prime}, z^{\prime}}\right)=\int_{--}^{\infty} \Psi_{0}^{\prime}\left(x^{\prime}, z^{\prime}\right) \exp \left(j k_{2}, x^{\prime}\right) d x^{\prime} \\
\Psi_{0}^{\prime}\left(x^{\prime}, z^{\prime}\right)=\frac{1}{2 \pi} \int_{-}^{-} \Psi_{0}^{\prime}\left(k_{;} ; z^{\prime}\right) \exp \left(-j k_{k_{0}}, x^{\prime}\right) d k_{x^{\prime}}
\end{gathered}
$$

Note that $\mathbf{k}_{\mathbf{x}^{\prime}}=\mathbf{k}_{\mathbf{\alpha}} / \cos \phi_{\mathrm{B}} \approx \mathbf{k}_{\mathbf{x}}$. After some more algebra one can easily show that,

$$
\Psi_{\circ}^{\prime}\left(k_{z} z^{\prime}\right)=\Psi_{0}^{\prime}\left(k_{\alpha} ; z\right) \exp \left(j k_{\alpha} \phi_{B} z\right) \text {. }
$$

Eqs. $(24,25)$ then transform to

$$
\begin{gathered}
\Psi_{\infty \alpha z}^{\prime}=j\left[k_{x}^{2} /\left(2 k_{0}\right)\right] \Psi_{\infty}^{\prime}-j E \Psi_{\alpha-1)}^{\prime} \\
\Psi_{\alpha(-1) z^{\prime}}^{\prime}=j\left[\left(k_{x^{\prime}}^{2}-4 k_{x^{\prime}}, k_{0} \Phi_{B}\right) /\left(2 k_{0}\right)\right] \Psi_{\alpha(-1)}^{\prime}-j D \Psi_{\infty \infty}
\end{gathered}
$$

where $\Psi_{\infty 0,-1}^{\prime}$ is the Fourier transform of $\Psi_{\infty,-1}^{\prime}$, defined through Eq. $(28,29)$. To derive the interaction transfer functions in the presence of propagational diffraction from Eqs. $(28,29)$. To derive the interaction transfer functions in the presence of propagational diffraction from Eq. (30), we normalize $z^{\prime}$ according to $\zeta^{\prime}=z^{\prime} / L$ and recast (30) in terms of $\Gamma_{00,1}^{\prime}\left(k_{x^{\prime}}, \zeta^{\prime}\right)=$ $\Psi_{\infty 0,-1}\left(\mathrm{k}_{\mathrm{C}^{\prime}} ; \mathrm{z}^{\prime}\right), \alpha$ and $\mathrm{Q}$. The constant $\alpha=\mathrm{k}_{0} \mathrm{CAL} / 2$ represents the peak phase delay of light through the sound cell with $\mathrm{A}=$ $A^{*}$. ( $C$ and $A$ are defined in respect of Eqs. (12 and 16.) $Q=2 \pi \lambda_{0} L / \Lambda_{0}$ is Klein-Cook parameter. We then solve the normalized equations using, for instance, the Laplace transform technique. $\Gamma_{\infty, 1}^{\prime}\left(k_{x^{\prime}}, \zeta\right)$ can finally be expressed in terms of the spectrum of the incident optical field (at $\mathrm{z}=0) \Gamma_{\text {inc }}^{\prime}\left(\mathrm{k}_{\mathrm{x}}\right)=\Psi_{\text {icc }}\left(\mathrm{k}_{\mathrm{\alpha}}\right)$.

$$
\begin{aligned}
& \Gamma_{\infty}^{\prime}\left(k_{x}, \zeta^{\prime}\right)=\Gamma_{\text {tec }}^{\prime}\left(\mathbf{k}_{x}, H_{0}^{\prime}\left(k_{x}, \zeta^{\prime}\right),\right. \\
& \Gamma_{\alpha-1)}^{\prime}\left(\mathbf{k}_{\alpha}, \zeta^{\prime}\right)=\Gamma_{\text {inc }}^{\prime}\left(\mathbf{k}_{\alpha}\right) \mathbf{H}_{-1}^{\prime}\left(\mathbf{k}_{\alpha}, \zeta^{\prime}\right),
\end{aligned}
$$

where $H_{0}^{\prime}\left(k_{x}, \zeta^{\prime}\right)$ and $H_{-1}^{\prime}\left(k_{x^{\prime}}, \zeta^{\prime}\right)$ are the interaction transfer functions of the zero and minus one orders expressible as

$$
\begin{aligned}
& \mathrm{H}_{0}^{\prime}\left(\mathrm{K}_{x^{\prime}}, \zeta^{\prime}\right)=\left(\exp \left[j\left(\mathrm{k}_{x}^{2} \mathrm{~L} /\left(2 \mathrm{k}_{0}\right)-\mathrm{k}_{\mathbf{x}}, \mathrm{Q} \Lambda_{d}(4 \pi)\right) \zeta^{\prime}\right]\right) \\
& \text { - }\left\{\cos \left[\left(\left(k_{\alpha}, Q \Lambda_{d} d(4 \pi)\right)^{2}+(\alpha / 2)^{2}\right)^{1 / 2} \zeta^{\prime}\right]\right. \\
& +\left(j k_{\alpha} Q \Lambda_{0} / 4 \pi\right)\left[\left(\left(k_{\alpha}, Q \Lambda_{0}((4 \pi))^{2}+(\alpha / 2)^{2}\right)\right]^{-1 / 2}\right. \\
& \left.\cdot \sin \left[\left(k_{\chi^{\prime}} Q \Lambda_{d}((4 \pi))^{2}+(\alpha / 2)^{2}\right)^{1 / 2} \zeta^{\prime}\right]\right\}
\end{aligned}
$$




$$
\begin{aligned}
& H_{1}^{\prime}\left(k_{x^{\prime}}, \zeta^{\prime}\right)=\left(\exp \left[j\left(k_{\alpha}^{2}, L /\left(2 k_{0}\right)-k_{x}, Q \Lambda_{d}(4 \pi)\right) \zeta^{\prime}\right]\right) \\
& \left.\cdot(-j \alpha / 2)\left[\left(k_{\alpha}, Q \Lambda_{0}(4 \pi)\right)^{2}+(\alpha / 2)^{2}\right)^{-1 / 2}\right] \\
& \cdot \sin \left[\left(\left(k_{\alpha^{\prime}}, Q \Lambda_{\delta}(4 \pi)\right)^{2}+(\alpha / 2)^{2}\right)^{1 / 2} \zeta^{\prime}\right]
\end{aligned}
$$

where $\Lambda_{0}$ is the wavelength of sound. Note that the above two solutions are derived in the new coordinate system ( $\left.x^{\prime}, \zeta^{\prime}\right)$. For the zero order, the $\mathrm{H}_{0}\left(\mathbf{k}_{\mathrm{x}^{\prime}}, \zeta\right)$ is the required transfer function in the nominal direction of the zero order diffracted light. However, for the minus one order, $\mathrm{H}_{1}\left(\mathrm{k}_{\alpha^{\prime}}, \zeta^{\prime}\right)$ should really be expressed along the nominal direction of the minus one order diffracted light instead of the direction of the zero order. This transformation can easily be done by defining a coordinate system which is at an angle of $-\phi_{B}$ to the $(x, z)$ coordinate system. ${ }^{3}$

\subsection{Simplified Treatment of Phase Confugation}

In an earlier section we have discussed the reconstruction of a hologram. Because of the close connection between degenerate four-wave mixing (DFWM) or phase conjugation and holography one can use the knowledge of one phenomenon (say phase conjugation) to have qualitative understanding of the other (say holography). The reference and viewing waves (in holography) serve the role of "pumps" during phase conjugation while the object field provides the probe beam. In what follows, we will show that using $\mathbf{k}$-space formalism students can construct simple geometrical figures and thus visualize the nature of phase conjugation without going through any complicated mathematics. ${ }^{4}$ An example of this approach is described below.

DFWM in collinear geometry when the probe has an amplitude and/or phase profile and one of the pumps is pulsed has been theoretically investigated by Bakos et al. ${ }^{5}$ Their analytical results can be readily duplicated using our simple k-space formalism, as discussed below. Fig. 5a shows the possible four-wave interactions where we have represented the probe by lines $\mathrm{AB}, \mathrm{A}^{\prime} \mathrm{B}^{\prime}$ and $\mathrm{A}^{\prime \prime} \mathrm{B}^{\prime \prime}$ to accomodate its amplitude and/or phase profile. Similarly, we have represented the backward traveling pump by lines $C D, C D^{\prime}$ and $C D^{\prime \prime}$ to illustrate its pulsed nature. From the figure, line segments $D A, D^{\prime}, D^{\prime} A^{\prime}, D^{\prime \prime} A^{\prime}, D^{\prime \prime}$, $D^{\prime} A^{\prime \prime}$ and $D^{\prime \prime} A^{\prime \prime}$ represent all possible contributions to the conjugate. As evident from the figure, the conjugate will contain an amplitude and/or phase profile in addition to being pulsed at the same time. This is because the constituent k-vectors of the conjugate have different directions and lengths. In order to quantify our physical arguments, we use simple geometry to predict the nature of the conjugate in terms of the characteristics of the probe and the pump. To this end, we refer to Fig. 5b which depicts a typicsl situation where the probe has an amplitude and/or phase profile (represented by $\Delta \phi$ ) and where the pump is pulsed (represented by $\Delta \mathbf{k}$ ). CA represents the resulting conjugate and BE is the forward traveling pump. From geometry, $|\mathrm{AC}|=\mathrm{k}_{0}-\Delta \mathrm{k}+$ higher order terms, where $\mathrm{k}_{0}$ is the nominal propagation constant for all four interacting waves. Thus, $(\Delta \mathbf{k})_{c} \Delta|A C|-k_{0} \sim-(\Delta k)$. Also, CAD $\Delta(\Delta \phi)_{c}=\Delta \phi+$ higher order terms. This implies that the conjugate will have wavefronts which are conjugated to the wavefronts of the probe, and the temporal spectrum of the conjugate $\left(\propto(\Delta k)_{c}\right)$ is of the same shape as the temporal spectrum of the pump. The negative sign in the relation $(\Delta \mathbf{k})_{c} \approx-(\Delta \mathbf{k})$ indicates coupling from the USB(LSB) of the pump to the LSB(USB) of the conjugate. This, in turn, implies hat the conjugate is approximately of the same duration as the pulsed pump. These findings are identical to the results in Bakos et al. ${ }^{5}$

\section{ILLUSTRATIVE DEMONSTRATIONS OF OPTICAL PROCESSING}

\subsection{An Acousto-optic Hybrid Bistable Device}

A simple demonstration of bistability and hysteresis amenable to classroom presentation is the hybrid acousto-optic bistable device, as shown in Fig. 6 . The diffracted light is detected by the photodetector of the feedback circuit. The feedback comprises a photodetector and a variable scaling adder. In order to show the hysteretic behavior of the system, a unipolar sawtooth signal with a peak-to-peak voltage of 5 volts at a typical frequency of $1 \mathrm{kHz}$ is injected into the scaling adder after the input buffer stage. This adds in phase with the detected signal from the photodetector and, as shown in Fig. 6, modulates the $40 \mathrm{Mhz}$ signal of the frequency generator which, in turn, drives the transducer in the AO cell. We may mention that after the detector circuit, a high-pass filter is employed to block any spurious DC voltage and noise signals. The DC blocking is required since it otherwise impedes the useful gain of the feedback signal in the OP AMP amplifier. 
For an operating frequency of $40 \mathrm{Mhz}$ for the sound, the effective $\mathrm{Q}$ of the cell, which is a figure of merit for defining the operating regime of the AO device, works out to 10 , which signifies the so-called near-Bragg regime. This means that, strictly speaking, higher order diffraction would occur. Fig. 7 shows the detected output $V_{D}$ from the first order and modulation on $\alpha_{0}$ as a function of time, with the corresponding hysteresis curves $V_{D}$ vs. $\hat{\alpha}_{o}$ for increasing values of the feedback parameter $B$, which was varied by changing the amplifier gain. For low values of $B$, there is no hysteresis as predicted from the Lissajous figure in Fig. 7a. A slight wrapping (or figure of eight) was observed in the Lissajous pattern because of distortion in the output owing to finite-amplitude inputs. As B increases, hysteresis develops, resulting in clipping of the output. The exact points denoting transitions in the output (see Fig. 7c) are determined by the threshold values on the corresponding hysteresis plots. Notice the asymmetry in the output with respect to the input due to the upper and lower thresholds of hysteresis. Fig. 8 shows a plot of the width of the hystersis curves, measured as the maximum between the up and down transitions, as a function of the feedback parameter $B$. Results depict a linear region in which the device could be operated as a programmable thresholding device.

Note that the output of the first-order diffracted light is approximately given by (see Eq. (22))

$$
\Psi_{1}=-j \psi_{\text {mc }} \sin (\hat{\alpha} / 2)
$$

where $\alpha$ represents the peak phase delay of the light through the Acoustooptic cell. Now,

$$
\varepsilon=\delta_{0}+\tilde{\beta}\left|\phi_{1}\right|^{2}
$$

where $\alpha_{0}$ is the external drive (or bias) and $B$ is the product of the gain constant of the amplifier and the conversion efficiency of the photodetector. The steady state output intensity is given by

$$
y=\sin ^{2}\left[\left(\hat{\alpha}_{0}+\tilde{\beta} y\right) / 2\right]
$$

where $y=\left|\psi_{1}\right|^{2}$ and where we have assumed $\psi_{\text {inc }}=1$ for convenience. It can be easily shown that when $B>2$, the steady-state curve assumes infinite gradients. That this corresponds to the onset of hysteresis can be shown from the rigorous theory of dynamical systems. Details are summarized in Ref. [6].

\section{2 z-scan Measurement of $\mathbf{n}_{2}$}

In materials which are cubically nonlinear, a Gaussian beam self-focusses or self-defocusses depending on the sign of the nonlinearity. This property can be used elegantly in a so-called z-scan measurement of the sign and magnitude of the nonlinearity, characterized by a nonlinear refractive index coefficient $n_{2}$. In this measurement method, a Guassian beam is initially focussed by an external lens into a nonlinear sample, and the far field diffraction pattem is observed, as shown in Fig. 9. By scanning the nonlinear sample across the focal plane of the external lens and observing the far-field spot size, one can infer the sign and value of the cubic nonlinearity $n_{2}$.

In a "thick" sample, the calculation of the far-field spot size as a function of the sample position with respect to the back focal plane of the external lens is rather involved. We will briefly outline the steps here as follows. First, a differential equation for the change of the q-parameter (defined earlier) with propagation in the nonlinear sample is derived by considering the sample as a collection of consecutively located thin slices. This equation reads

$$
d q / d z=1+\left[4 n_{2} a^{2}(z) / n_{0} w^{2}(z)\right] q^{2}
$$

where the first term on the RHS accounts for propagational diffraction and the second term is due to the induced lensing effect, and where $\mathrm{a}(\mathrm{z})$ and $\mathrm{w}(\mathrm{z})$ are the on-axis amplitude and width of the beam. The variation of the Gaussian beam width is next found from (36) in the nonlinear sample, given a set of appropriate initial conditions, commensurate with the focussing effect of the external lens. The exit width and radius of curvature are then determined, and the far-field width thus calculated. Fig. 10 shows the variation of the width of the beam in the far-field observation plane for positive and negative nonlinearities. Note that the sign of the nonlinearity can be determined from the gradient of the curve(s) around the back focal plane of the external lens for a thin sample, and around the point where the curves intersect the $\mathrm{n}_{2}=0$ curve for a thick sample. Details can be found in Ref. [7]. 
We conducted experiments with a saturated solution of chlorophyll in ethyl aclohol as the nonlinear medium in a curvette of thickness $1 \mathrm{~cm}$ along the direction of propagation of the Gaussian beam. A $10 \mathrm{~mW} \mathrm{CW} \mathrm{He-Ne} \mathrm{laser} \mathrm{(wavelength}$ $633 \mathrm{~nm}$ ) with a beam diameter of $0.77 \mathrm{~mm}$ was used as the source. A lens of focal length $5 \mathrm{~cm}$ was used to focus the beam into the nonlinear material. Fig. 11 shows a plot of the beamwidth measured at a distance of $1 \mathrm{~m}$ from the exit face of the cell, as a function of the lens-sample separation. The nonlinear refractive index is clearly negative in sign, and its exact value can be computed from our derived theory to be $-2.4 \times 10^{-13} \mathrm{~m}^{2} / \mathrm{V}^{2}$. The simplicity of measurement (probably not the analysis) makes the experiment conducive to a ready classroom demonstration.

\section{OPTICAL LOGIC OPERATIONS USING TRANSPARENCIES}

In digital optics, data are represented by ones and zeros just as in digital electronics. The binary data form the basis for numeric and logical operations. Among the set of logical operations are exact match, less than, greater than, less than or equal to, greater than or equal to, maximum, minimum and others. These operations are useful for higher level functions as well, such as those found in database management.

There are many ways to represent ones and zeros in optical form and these include intensity, phase, polarization, shadow casting and others. In what follows we will use a form of shadow casting since we use a parallel light source (the transparency projector) and illustrate the performance of logic operations with common transparencies. We provide two examples of how transparencies can be used to help in the educational process. The first concerns matching such as one would do in searching for a variety of words in common text such as books, newspapers, articles, etc. The second concerns the execution of optical content addressable memory operations.

\subsection{Character and Word Matching}

The optical arrangement shown in Fig. 12, can be used to implement the logic equation

$$
\mathbf{A} \overline{\mathbf{B}}+\overline{\mathbf{A}} \mathbf{B}=\mathbf{0} .
$$

When this equation is satisfied; A = B where A and B are individual characters or words. The presense or absense of light is used to represent the data in binary form. In order to perform exact match comparisons one or more search arguments are loaded into spatial light modulator 2 (SLM-2). Data to be searched are horizontally stepped through SLM-1 and matches are detected when no light is received at the photo detector array thus satisfying the above equation. To perform operations on many characters or words simultaneously one need only expand the size of the SLM's and introduce a few control characters.

In the classroom environment the transparency projector serves as the light source. The search argument(s) is represented by columns of squares that are transparent or opaque to represent the binary data. For instance, to represent the name "Boris", five contiguous columns of light and dark squares would be required, ignoring any control characters. Each of the columns would contain a unique coding for that character and its complement since the above equation is based upon a character and its complement. The data to be searched would be represented in a similar way. In order to perform a search the transparency with the term "Boris" on it (the contents of SLM-2) is held stationary. The transparency with the data to be searched on it (the contents of SLM-1) is stepped across the stationary transparency one character at a time. If the term "Boris" is in the data, then when it is aligned with the search transparency no light will pass through the transparencies and the match will have occurred. This set up also illustrates some of the problems that must be solved in optical systems such as alignment, light leakage, etc.

\subsection{Optical Content Addressable Memories}

Electronic content addressable memories (CAM) have long been useful for performing various logic and searching operations. Shown in Fig. 13 is the structure of a CAM. The data to be operated upon are stored in the CAM array and the search argument(s) is stored in the comparand register. The mask register is used to select those columns of data that are of interest to the current search operation. The response registers are used to store intermediate and final results. The significant aspects of a CAM are that data are accessed by content and in parallel. 
The concepts discussed above apply equally well to optical CAM's. In our illustration we again use a shadow casting technique. The data are represented as pairs of pixels as shown in Fig. 14 with opposite representations for comparand and array data and $d$ is for don't care. Suppose we want to perform a variety of searches on the data shown in Fig. 15a. Here we will illustrate only exact match, greater than and less than as other searches are constructed from these basic operations. Shown in Fig. 15b is the representation of the data using the coding in Fig. 14. In our illustration we will concentrate on the data in Fig. $15 \mathrm{c}$ to which two response registers have been added. The two response registers are used to obtain the results of the search and will be explained later.

Returning to the use of transparencies, the first transparency that is placed on the projector represents the array data of Fig. 15b. Since the CAM performs parallel operations, the comparand contents are replicated as many times as there are words (rows) in the array. Thus, the data in the array of Fig. 15c contains the data in the array of Fig. 15b overlayed with another transparency that replicates the data in the comparand register. Note that if a particular row is completely dark; then the comparand data match the data in that row.

In order to provide more rigor in illustrating the concepts, we must proceed one column at a time and use the two response register columns. For this we need two don't care strips, a short one and a long one for each row in the array. A don't care strip consists of don't care symbols in each cell. Thus, if a don't care strip is placed over a row, all the light will be blocked. The short one covers the entire row plus the first response register and the long one covers the entire row plus both response registers. Returning the Fig. 15c to illustrate exact matches, we first consider column 1 . Rows 3 and 5 have light so the long don't care strip is used and removes them from further consideration. Then we consider column 2; similarly this removes row 4. Since there is light coming from column 3, row 2, the long don't care strip is used to eliminate row 2. Since all columns have been considered and light is emanating from the second response register of row 1 then that represents a hit. Thus, the data in row 1 exactly match the data in the comparand register.

We now consider finding those rows in the array which are greater than the value in the response register. To implement this search we will use both don't care strips. Considering column 1, it is clear that light is emanating from the one portion of the pixels (the left side) of rows 3 and 5. This indicates that we now know that the data contained in these rows is greater than the value contained in the comparand register. So we use the short don't care strip to cover rows 3 and 5 . This removes them from further consideration and indicates positive responses by the presense of light in the second response register. Considering column 2, row 4 is removed from further consideration by being covered by the long don't care strip since light is emanating from the zero position. Finally, considering column 3 , we cover row 2 since light is emanating from the zero position. Since we have exhausted the columns, we must now examine the first response register for any remaining sources of light. If any remain, then their data must be equal to the search argument and the long don't care strip is used to cover the response registers. Thus, the results of the search indicate that rows 3 and 5 contain data that are greater than the data in the response register.

In a similar way, a less than search can be performed and that is left to the interested reader. With these three searches as a basis, it is clear that other more complex searches can be performed. While greater than or equal to and less than or equal to can be performed directly, other more complex seraches (e.g. between limits, etc.) will require intermediate storage of results.

\section{CONCLUSION}

In conclusion, we have presented, in brief, some of the novel classroom and laboratory teaching methods which we have been using in the area of optics. Enrollment in classes have increased particularily in Optical Information Processing, which had a class size of about nine in 1986 and about twenty-seven in 1990. We hope our approach and efforts will sustain the general interest in optics in the years to come.

\section{REFERENCES}

1. P.P. Banerjee and T.C. Poon, "Principles of Applied Optics," Irwin, Boston, 1991.

2. A. Korpel, "Acousto-optics," Marcel-Dekker, New York, 1988. 
3. P.P. Banerjee and C.W. Tarn, "A fourier Transform Approach to Acoustooptic Interactions in the Presence of Propagational Diffraction," ACUSTICA, Vol. 74, pp. 181-191, 1991.

4. P.K. Ghosh and P.P. Banerjee, "A Simple Geometrical Approach to Study Degenerate Four Wave Mixing in a Cubically Nonlinear Medium," PIERS, 1991.

5. J.S. Bakos, G.P. Djotjan and T. Juhanz, "Phase Conjugation by four-wave Mixing witha Nonstationary Pumping Wave," J. Opt. Soc. Am. B, Vol. 6(B0), pp. 1540-1543, 1989.

6. P.P. Banerjee, "Bistability and Chaos in Acousto-optics,"Fourth School on Acousto-optics, Golansk, Poland, 1989.

7. P.P. Banerjee, R.M. Misra and M. Maghraoui, "Theoretical and Experimental Studies of Propagation of Beam Through a Finite Sample of a Cubically Nonlinear Material," J. Opt. Soc. Am. B, Vol. 8, No. 5, pp. 1072-1080, 1991.

8. P.B. Berra, A. Ghafoor, P.A. Mitkas, S.J. Marcinkowski and M. Guizani, "The Impact of Optics on Data and Knowledge Base Systems," IEEE Transactions on Knowledge and Data Engineering, Vol. 1, No. 1, pp. 111-132, 1989.

9. P.S. Guilfoyle and W. Jackson Wiley, "Combinatorial Logic Based Optical Computing," Proceedings of SPIE, Vol. 639-17, 1986. 


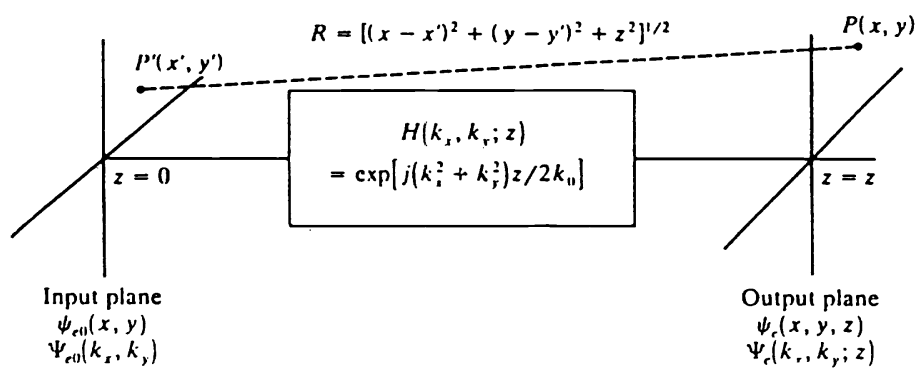

Fig 1 Block-diagrammatic representation of the spatial transfer function of propagation. The input and output planes have primer! and unprimed coordinate systems, respectively.

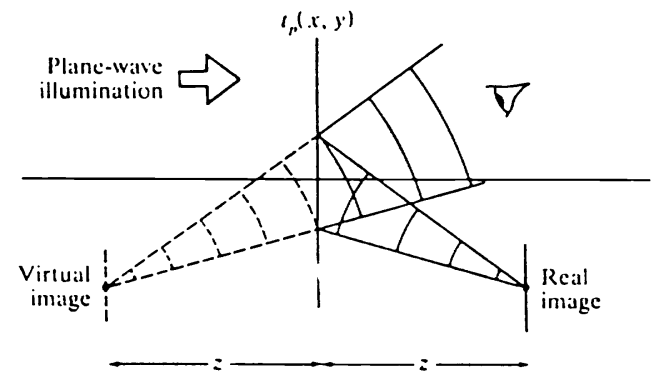

Fig 2 Reconstruction from the Lcith-Upatnciks hologram using a plane wave.

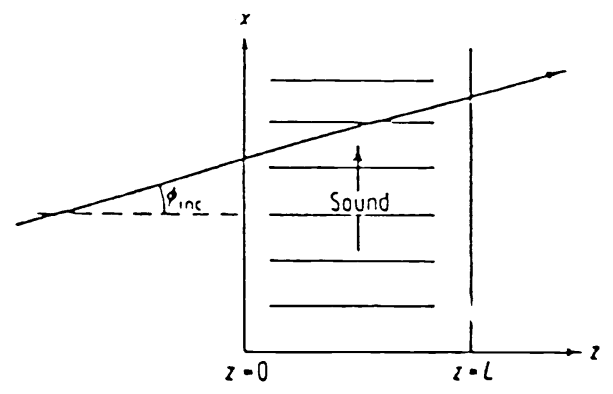

Fig 3 Conventional sound-light interaction configuration

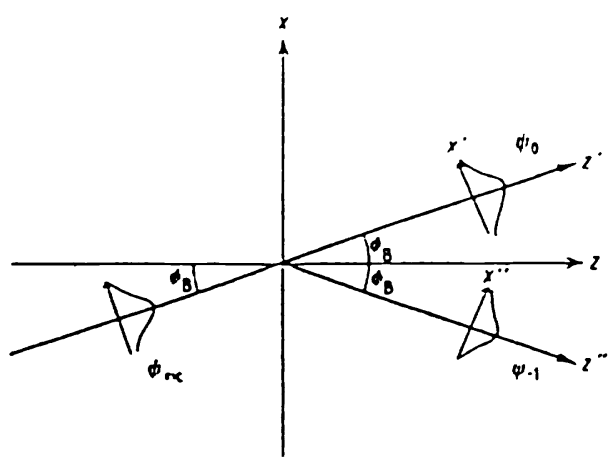

Fig. 4 Difraction geometry and coordinate systems used in defining angular spectra.
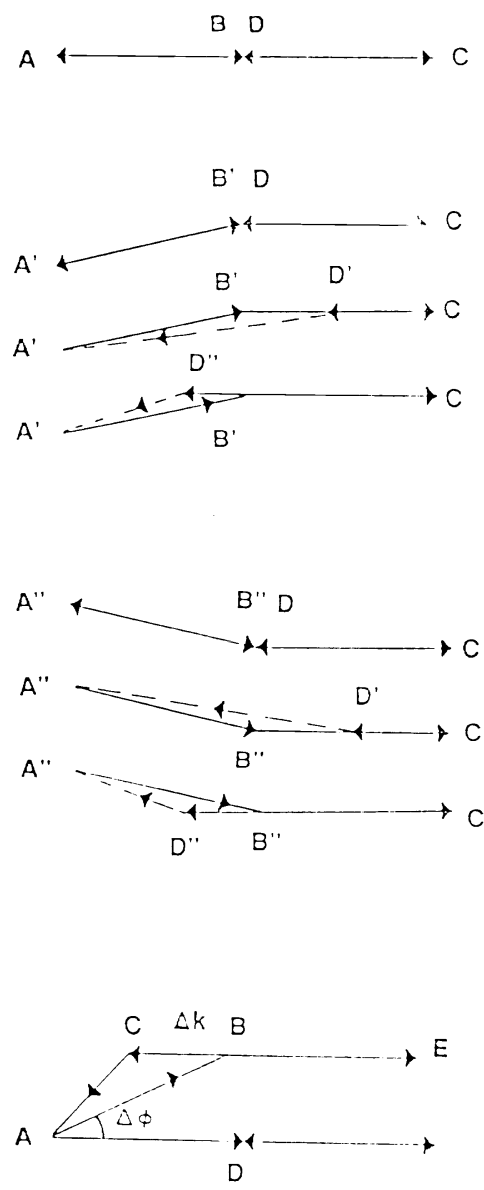

Fig 5 (a) Possible four-wave interactions in nominally collinear geometry when the probe has an amplitude/phase profile and the backward traveling pump is pulsed; (b) Typical geometry to calculate $(\mathrm{K})_{\mathrm{C}}$ and ()$_{\mathrm{C}}$. The lengths $\mathrm{AB}, \mathrm{BE}$ and $\mathrm{AD}$ are each equal to $\mathrm{k}_{0}$. 


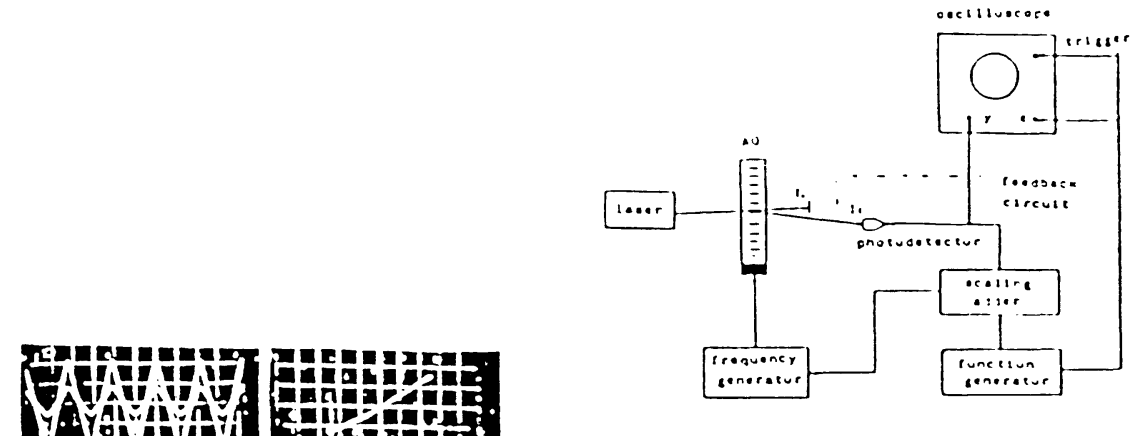

Pig. 6 Experiolental setup to observe

far-ficid aringinat

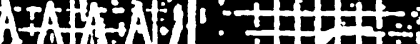

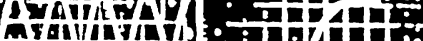
hysteresis and bistability.

Pig. 7 CRT traces of the input and output waveforms monitored as a function of time together with the Lissajous patterns. The sequence $(a)-(c)$ is for increasing values of the feedback.

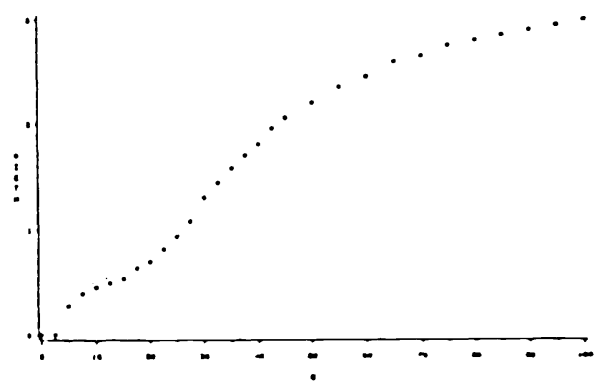

Fig. 8 Variation of the width of the hysteresis curve as a function of the feedback parameter for first-order detection.

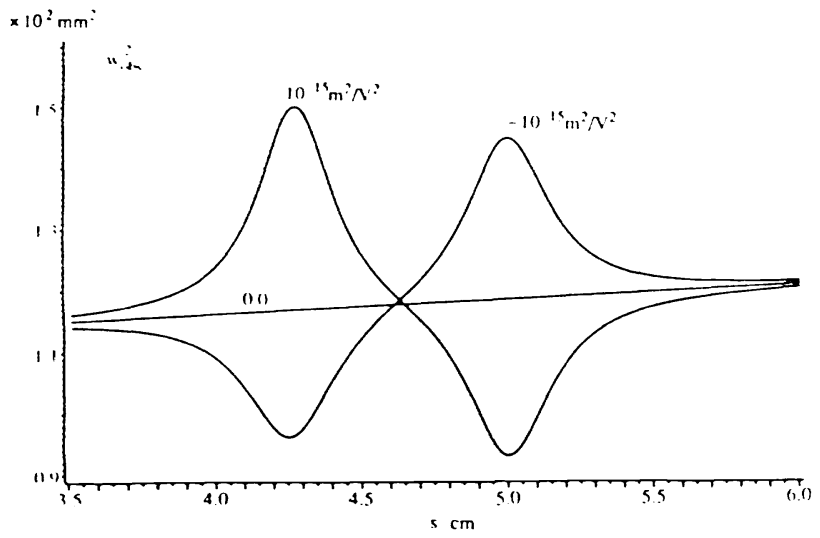

Fig. 10 Plot of the square of the far-field observation width at a distance of $1 \mathrm{~m}$ from the exit face of the sample versus the lens-sample separation distance $s$ with $n_{2}$ as a parameter. $\quad\left(\lambda_{0}=\right.$ $640 \mathrm{~nm}, f_{0}=5 \mathrm{~cm}, z_{\text {exit }}=1 \mathrm{~cm}, P=10 \mathrm{~mW}, n_{0}=1.36$, and the laser-beam-intensity diameter is $0.77 \mathrm{~mm}$ ).

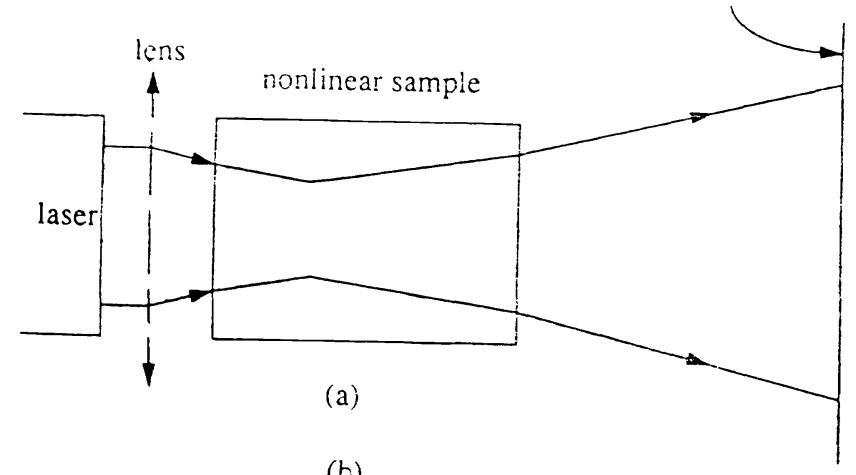

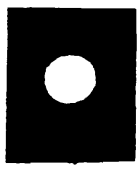

(i) (b)

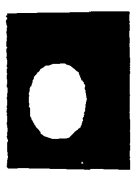

(ii)

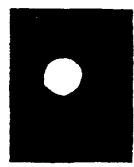

(iii)
Fig. 9 (a) Experimental setup for nonlinear refractive-index measurement. (b) Far-field diffractions (i) without the nonlinear sample, (ii) with light focused in front of the sample, (iii) with light focused within the nonlinear sample close to the exit face.

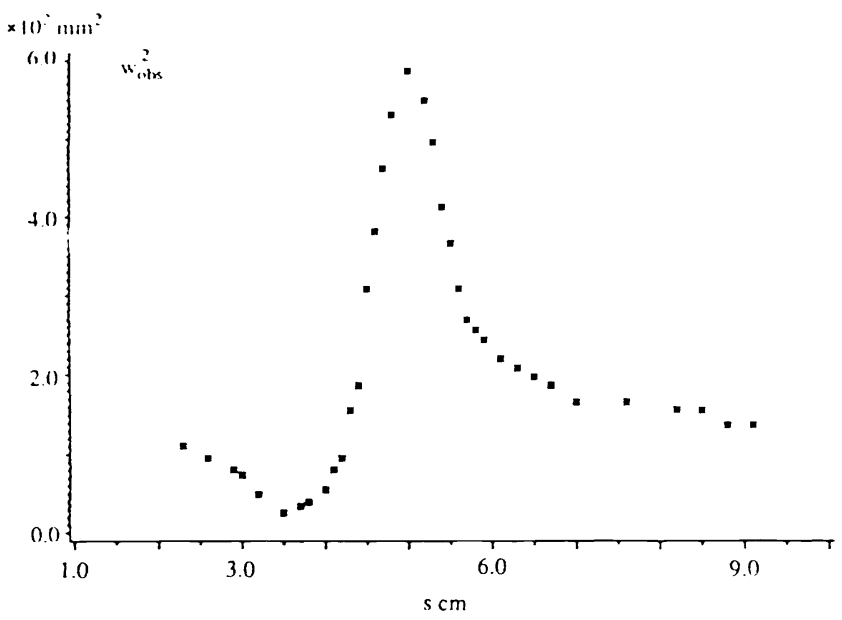

Fig. If Plot of the square of the far-field observation width at a distance of $1 \mathrm{~m}$ from the exit face of the chlorophyll-ethanol sample versus the lens-sample separation distance $s\left(\lambda_{0}=640 \mathrm{~nm}\right.$, $f_{0}=5 \mathrm{~cm}, z_{\text {exit }}=1 \mathrm{~cm}, P=10 \mathrm{~mW}, n_{0}=1.36$, and the laserbenm-intensity diameter is $0.77 \mathrm{~mm}$ ). 


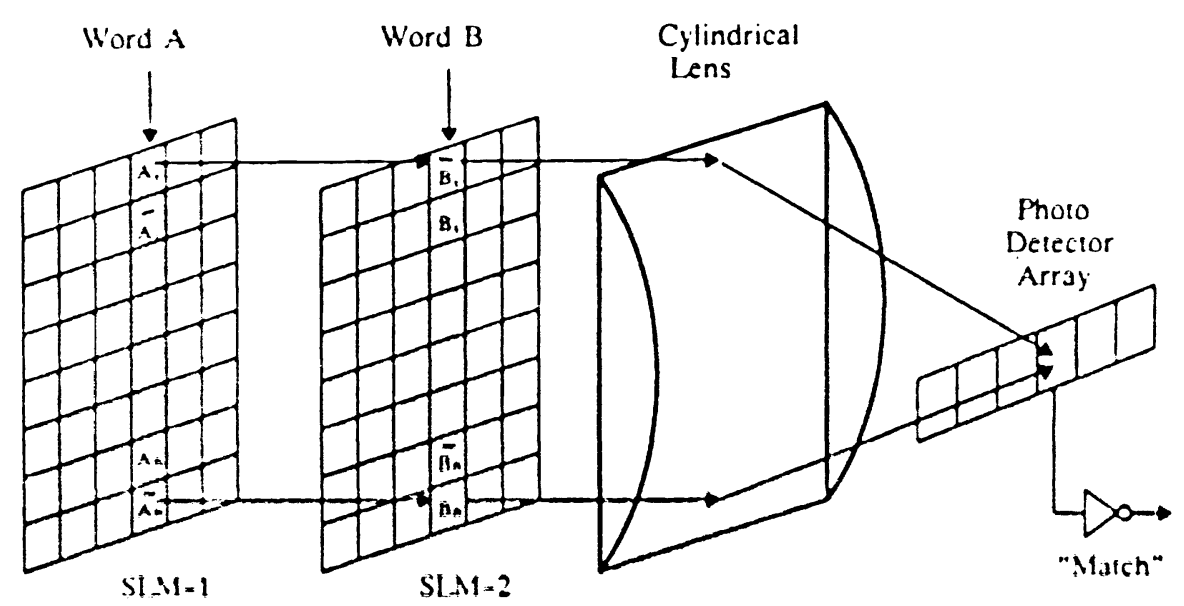

Fig. 12 Optical arrangement for character matching.

\begin{tabular}{|c|c|c|c|}
\hline & 0 & 1 & $d$ \\
\hline $\begin{array}{l}\text { Comparan } \\
\text { Rejlster }\end{array}$ & & & \\
\hline $\begin{array}{l}\text { CAM } \\
\text { Array A }\end{array}$ & & & \\
\hline
\end{tabular}

Fig. 14 Data coding in CAM. $c$

m

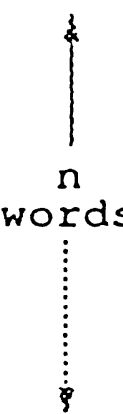

$k$
$n$
$\vdots$
$\vdots$
$\vdots$
$\vdots$

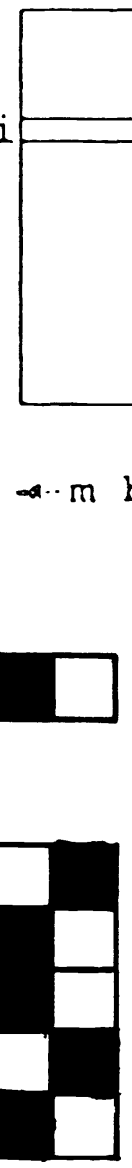

(b)

Comparand register Mask register

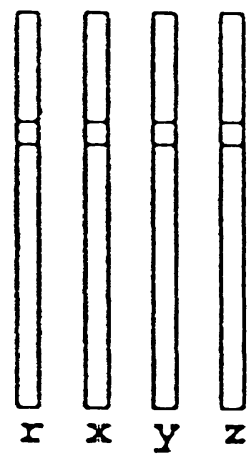

j

CAM array

Fig. 13 Content addressable memory.

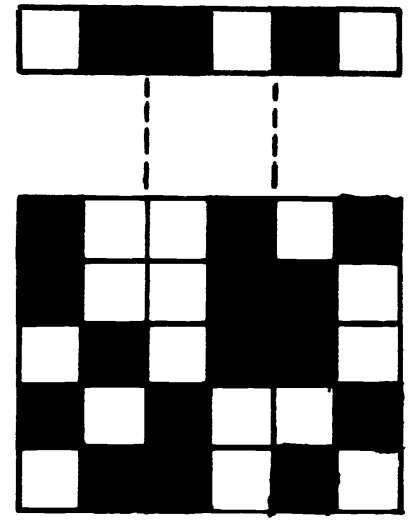

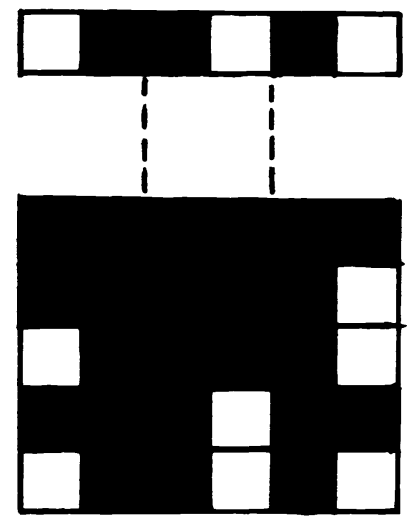
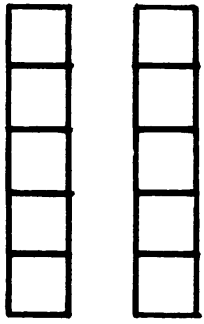

(c)
Response store registers

Fig. 15 Optical searches. 